\title{
Custo de vida comparativo das regiões metropolitanas brasileiras: 1996-2014
}

\author{
Alexandre Nunes Almeida \\ Professor - Depto. de Economia, Administração e Sociologia - Escola Superior de Agronomia Luiz \\ de Queiroz - (ESALQ/USP) - Endereço: Av. Pádua Dias, 11 - Piracicaba - São Paulo \\ CEP: 13418-000 - E-mail: alex.almeida@usp.br
}

Carlos Roberto Azzoni

Professor - Depto. de Economia - Faculdade de Economia, Administração e Contabilidade - (FEAVUSP)

Endereço: Av. Prof. Luciano Gualberto, 908 - Butantã - São Paulo

CEP: 05508-010 - E-mail: cazzoni@usp.br

Recebido em 29 de janeiro de 2014. Aceito em 11 de setembro de 2015.

\section{Resumo}

Neste trabalho estimamos os níveis de custo de vida de 11 regiões metropolitanas brasileiras no período 1996-2014. Aplicamos o método Country Product Dummy (CPD) a dados das pesquisas de orçamentos familiares de 1996, 2003 e 2009. Interpolamos os pesos orçamentários para os anos intermediários e usamos dados de índices de preços regionais para interpolar e atualizar os preços. Os resultados indicam que os maiores custos de vida estão em Brasília, São Paulo e Rio de Janeiro, sendo que Goiânia apresentou o maior crescimento relativo no período.

\section{Palavras-Chave}

Níveis de custo de vida. Regiões metropolitanas. Country Product Dummy.

\begin{abstract}
We compute cost of living levels for 11 Brazilian metropolitan regions in the period 1996-2014. We apply the Country Product Dummy (CPD) method to data from the household expenditure surveys of 1996, 2003 and 2009. We calculate the levels for these years, and use data from regional price indexes to interpolate the results for years in-between. The results indicate that Brasília, São Paulo and Rio de Janeiro are the most expensive metropolitan areas, and Goiânia presented the largest growth.
\end{abstract}

\section{Keywords}

Cost of living levels. Metropolitan areas. Country Product Dummy.

\section{JEL Classification}

R. R32.

\footnotetext{
-Agradecemos os comentários de dois pareceristas anônimos, que contribuíram substancialmente para a melhoria do trabalho. Erros remanescentes são de responsabilidade exclusiva dos autores.
} 


\section{Introdução}

O país vive um momento econômico bastante distinto daquele de décadas passadas. Com a implementação do plano real em 1994 e a estabilidade da moeda, novas gerações de indivíduos experimentaram um quadro econômico até então inédito no país. Esse aspecto foi e é de importância relevante na vida das famílias, dado que o controle sustentado da inflação permite ao chefe de família planejar por prazos maiores seu orçamento doméstico em função da renda obtida, melhorando o bem-estar.

Além da estabilidade econômica, outros fatores, como as reformas no sistema educacional, o aumento do emprego formal, a apreciação do valor do salário mínimo e, mais recentemente, a massiva expansão de programas de transferência de renda, como Bolsa Família e Benefícios de Prestação Continuada, têm contribuído significativamente para a redução da desigualdade de renda e redução da pobreza (Neri, 2010). Não se pode negligenciar também a abertura da economia durante os anos 90, que possibilitou acesso a novas variedades e marcas de produtos, com potencial efeito sobre o conjunto de bens à disposição dos consumidores. Adicionalmente, observou-se crescimento substancial da oferta agrícola, decorrente de significativos ganhos de produtividade, o que alterou substancialmente alguns preços relativos, principalmente os de alimentos (Azzoni et al., 2009).

Dados esses fatores de interesse para todas as regiões, deve-se levar em conta o conhecido fato de que existe uma grande disparidade regional nos níveis de renda e bem-estar, assim como nos níveis de custo de vida entre regiões e cidades (Azzoni et al., 2003). Terá todo o movimento ocorrido no país em termos de consumo afetado o diferencial regional de custo de vida observado no passado? A ascensão das classes mais pobres na escala de consumo, com o alargamento da base da pirâmide, certamente afetou a composição da cesta típica de bens de todas as regiões, assim como pode ter afetado preços relativos, notadamente pela maior procura por bens de salário. Da mesma forma, o aumento da participação do setor terciário na economia e o envelhecimento da população podem ter tido efeitos importantes em termos de mudanças no padrão de consumo. Considerando-se que esses movimentos apresentaram intensidades variáveis em distintas regiões, é possível que os efeitos tenham sido distintos, podendo levar a modificações nos diferenciais de custo de vida observados anteriormente. 
$\mathrm{Na}$ literatura em geral, a importância da existência de um índice de custo de vida entre cidades é enfatizada em vários trabalhos, tais como Deller et al. (1996), Walden (1997), Johnston et al. (1996), McMahon (1991), Aten (2005, 2007, 2008), Aten e D'Souza (2008), Aten et al. (2011a, 2011b, 2012), Aten e Reinsdor (2010), Navamuel et al. (2012), Morollón et al. (2013). Esse tema foi tratado pioneiramente no Brasil por Savedoff (1990), que discutiu com bastante propriedade a importância e as dificuldades em se obter um índice de custo de vida entre as cidades brasileiras. Nos Estados Unidos, o Bureau of Labor Statistics tem desenvolvido índices dessa natureza (Kokoski, 1993; Kokoski e Moulton, 1996). Azzoni, Carmo e Menezes (2000), Menezes e Azzoni (2003) construíram índices de custo de vida para as maiores regiões metropolitanas brasileiras, concluindo haver diferença importante nos níveis de custo de vida entre elas, diferença que se ampliou ao longo do período analisado (1981-1999). Menezes e Azzoni (2007) estimaram os custos relativos de aluguéis entre as capitais brasileiras, revelando os diferenciais existentes nos níveis desse item da despesa domiciliar.

Interessa pois averiguar se os resultados anteriormente obtidos se mantêm, ou se mudanças importantes ocorreram nos diferenciais de custo de vida entre as regiões metropolitanas brasileiras. Assim, o objetivo deste trabalho é atualizar os trabalhos de Azzoni et al. (2000) e Menezes e Azzoni (2003), em que se construíram índices de custo de vida para as nove regiões metropolitanas (RM), além de Goiânia e Distrito Federal, para anos das décadas de 1980 e 1990. O período de análise agora abrange o período 1996-2014, e se utilizam os preços disponibilizados pelo IBGE (IPCA), as Pesquisas de Orçamentos Familiares (POF) de 1996, 2003 e 2009, também do IBGE, e informações sobre aluguéis das PNAD.

Além da breve introdução, o artigo está divido em mais seis seções. Na próxima é apresentada a metodologia, seguindo-se a descrição dos dados utilizados, na terceira, e a apresentação dos índices calculados, na quarta. Na seção 5 avalia-se a possível existência de convergência nos níveis de custo de vida das RM. Na seção seguinte apresenta-se a evolução dos valores reais e da paridade do poder de compra das RM e verifica-se como tem evoluído a disparidade regional dessas duas formas de medir a renda regional. Finalmente, na última seção apresentam-se as considerações finais do estudo. 


\section{Metodologia}

Índices bilaterais comparam níveis de preços em dois momentos do tempo. O cálculo do nível de custo de vida relativo entre cidades exige índice muito mais complexo, pois deve necessariamente ser multilateral, comparando várias cidades simultaneamente (Diewert, 1988). Esse tema originou-se da necessidade de comparação da paridade de poder de compra entre países, em que despontaram os estudos de Deaton e Muellbauer (1980), Heston e Lipsey (1999) e Heston e Summers (1996). Conforme detalhadamente apresentado em Menezes (1999) e Navamuel et al. (2013), tal índice deve apresentar as propriedades de reversibilidade de região (a paridade de preço $\mathrm{P}_{\mathrm{A} / \mathrm{B}}$ da região $\mathrm{A}$ com respeito à região $\mathrm{B}$ deve satisfazer à condição $\mathrm{P}_{\mathrm{A} / \mathrm{B}}=1 / \mathrm{P}_{\mathrm{B} / \mathrm{A}}$ ) e de transitividade, ou circularidade (para três regiões quaisquer, $\mathrm{P}_{\mathrm{A} / \mathrm{B}}=\mathrm{P}_{\mathrm{A} / \mathrm{C}} / \mathrm{P}_{\mathrm{B} / \mathrm{C}}$ ). Essas condições são respeitadas pelos métodos EKS (Dreschler, 1973) e CPD (Country Product Dummy), desenvolvido por Kravis et al. (1982), como discutido em Azzoni et al. (2003). ${ }^{1}$

Como em Menezes (1999), Azzoni et al., (2000), Menezes e Azzoni (2001) e Menezes et al. (2007), neste trabalho utiliza-se o método Country Product Dummy. A Equação (1) é estimada empregando o método de Mínimos Quadrados Ponderados, em que os ponderadores correspondem aos pesos de cada item no dispêndio familiar. Para cada ano, a equação estimada é:

$$
\ln p_{i k}=\sum_{k=1}^{K} b_{k} x_{k i}+\sum_{j=2}^{J} \gamma_{j i} y_{j i}+e_{i k}
$$

em que:

$\ln p_{i k}=\log$ neperiano do preço do bem (item) i na cidade $k$

$x_{i k}(k=1 \ldots K)=$ variável binária igual a 1 se o preço do bem i foi coletado na cidade $k$ e zero nas demais cidades.

$y_{i j}(j=2 \ldots J)=$ variável binária igual a 1 se o bem i contém a j-ésima característica e zero nas demais.

$e_{i k}=$ variável aleatória com média zero e provavelmente com variância heterocedástica.

${ }^{1}$ Para uma análise extensa do assunto, ver Heston e Lipsey (1999) 
O coeficiente de $x_{i k}, b_{k}$, corresponde ao log neperiano do preço relativo entre as $k$ áreas. Sob as hipóteses do modelo, os antilog de $\hat{b}_{k}$ são estimadores consistentes e transitivos dos níveis de preços relativos, ou seja, são índices de preços entre as $k$ cidades, conforme discutido em Menezes (1999). Uma grande vantagem desse método é que, mesmo com ausência de alguns valores, possibilita estimar as equações com base nas informações disponíveis (Summers, 1977; Selvanathan e Rao, 1994; Navamuel et al., 2012).

\section{Dados}

Para a obtenção dos pesos de cada item nos orçamentos familiares, parte-se do microdados das três últimas edições da Pesquisa de Orçamentos Familiares (POF), realizadas pelo IBGE nos períodos outubro/1995-setembro/1996, julho/2002-junho/2003 e maio/2008-maio/2009. As POF levantam informações sobre condições do domicílio, como abastecimento de água, infraestrutura sanitária e número de cômodos, número de famílias (unidades de consumo) residindo no mesmo espaço domiciliar, e também as características dos indivíduos, como sexo, nível de instrução, idade, frequência à escola, peso, altura e posição da família (chefe, cônjuge, filho, outro parente, agregado, pensionista, empregado doméstico e parente do empregado doméstico); há também informações sobre as despesas com melhoria (reforma) do domicílio, bens duráveis, etc. De particular interesse para este estudo são as informações detalhadas sobre gastos com alimentação, higiene e limpeza, telefone, etc., além das informações sobre gastos mensais e recebimentos salariais e não salariais. Como as informações coletadas podem ter períodos de referência distintos, de sete dias até 12 meses, dependendo da natureza do registro, o IBGE disponibiliza fatores de anualização, que permitem que os valores sejam convertidos para valores anuais (IBGE, 2010).

As POF entrevistaram 16.013 unidades domiciliares em 1995/96, 48.470 em 2002/03 e 55.970 em 2008/09, sendo que a primeira cobriu apenas as nove regiões metropolitanas, Goiânia e Distrito Federal. Em função das variações dos preços nominais que ocorrem ao longo do período da coleta, geralmente 12 meses, o IBGE disponibiliza um deflator, referido à data de referência da pes- 
quisa pré-definida pelo Instituto. ${ }^{2}$ Com isso, é possível comparar e analisar os registros em valores reais de acordo com o mês base definido pelo IBGE. A definição das amostras respectivas partiu do Censo Demográfico de 1991, no caso da POF 1995/06, e do Censo Demográfico de 2000 para as duas últimas edições (IBGE, 2004; IBGE 2010). Os fatores de expansão baseiam-se na Contagem Populacional de 1996 e no Censo Demográfico de 2000. Para manter a homogeneidade da cesta de bens e serviços entre as regiões, atendendo às propriedades impostas pelo método $\mathrm{CPD}$, foi possível escolher 41 produtos (Quadro 1, no apêndice), com vistas a definir uma cesta minimamente aceitável quanto ao consumo de produtos e uso de serviços considerados essenciais pelos domicílios.

As POF constituem riquíssima fonte de informação, mas apresentam muitas limitações, naturais em levantamentos de tão complexa execução (IBGE, 2010). Observa-se elevada presença de despesas com valor zero, inclusive para itens de consumo frequente, como alimentos. Dois fatores concorrem para tanto. Em primeiro lugar, o gasto pode não ter sido realizado durante o período da entrevista, embora isto não queira dizer que o bem não tenha sido consumido, a partir de estoques feitos anteriormente. Em segundo, pode ser que o bem simplesmente não faça parte da cesta de consumo do domicílio, por hábito, renda etc. Para remediar o problema gerado pela ausência dessas informações, a solução foi agregar as famílias por classes de renda, uma prática bastante comum em estudos de demanda (Menezes et al., 2008). Assim, as famílias foram agregadas em oito classes de renda, de acordo com a despesa mensal total per capita. Em outras palavras, em cada cidade foram construídos oito domicílios representativos, que representam as médias dos domicílios inseridos nas respectivas classes de renda. Geraram-se assim 88 observações (8 classes de rendas vezes 11 localidades) para cada ano. Especificamente, foram usadas informações de 16.013 domicílios da POF 95/06, 6.573 de 2002/03 e 8.575 de 2008/09.

A partir das POF, identificaram-se os pesos dos 41 itens nos orçamentos das famílias representativas em cada cidade, nos anos de realização dos levantamentos, representados nos gráficos da Figura 1. Pode-se notar que os grupos com maiores pesos nos orçamentos familiares são habitação e alimentação, que, somados, representam

2 As datas de referência são 15 de setembro de 1996, 15 de janeiro de 2003 e 15 de janeiro de 2009. 
mais de 50\% dos gastos, em média. Observa-se também que a parcela despendida com habitação elevou-se entre 1996 e 2009 em praticamente todas as localidades, enquanto que a parcela destinada a gastos com alimentação, segundo lugar no ranking, reduziram-se no mesmo período. O grupo de despesas com transportes ocupa, para a maior parte das localidades, o terceiro lugar dentro do orçamento familiar. Observa-se que a participação deste grupo, com exceção de Recife, também se elevou entre 2003 e 2009. Os grupos de despesas com vestuário e saúde e despesas pessoais orbitaram entre o terceiro e quarto lugares no quadro geral. Finalmente, o grupo educação manteve-se no último lugar, variando entre 3\% e $8 \%$ do orçamento doméstico mensal. Para os anos intermediários, aplicou-se interpolação linear, admitindo que as mudanças obedeceram a um processo com essa característica. ${ }^{3}$ Para os anos posteriores a 2009, os pesos desse ano foram repetidos. Dessa maneira, as mudanças nos padrões de consumo entre 1996 e 2009 estão bem representadas, embora as mudanças posteriores não tenham sido incorporadas, por falta de informação. Todavia, como mudanças em padrão de consumo são lentas e graduais, acredita-se que essa limitação não compromete a utilidade dos resultados. ${ }^{4}$

3 Como as mudanças, por seu caráter estrutural, são naturalmente lentas, a opção por outro método (exponencial, por exemplo) produziria poucos resultados práticos.

4 Em todo cálculo de índice de custo de vida, a agregação dos itens de consumo é sempre uma decisão crítica, tendo em vista que, dependendo do índice utilizado, diferentes formas de agregar os itens podem levar a resultados distintos. A escolha feita no presente caso derivou de necessidades práticas, dada a limitação de informações. Todavia, dado que os mesmos critérios foram aplicados a todas as regiões metropolitanas, modificações nos níveis comparativos de custo de vida calculados somente apareceriam se as importâncias relativas de produtos e serviços tivessem variado diferentemente entre áreas, ao longo do tempo. Uma avaliação da evolução dos pesos dos vários itens não sugerem nada nessa direção. 

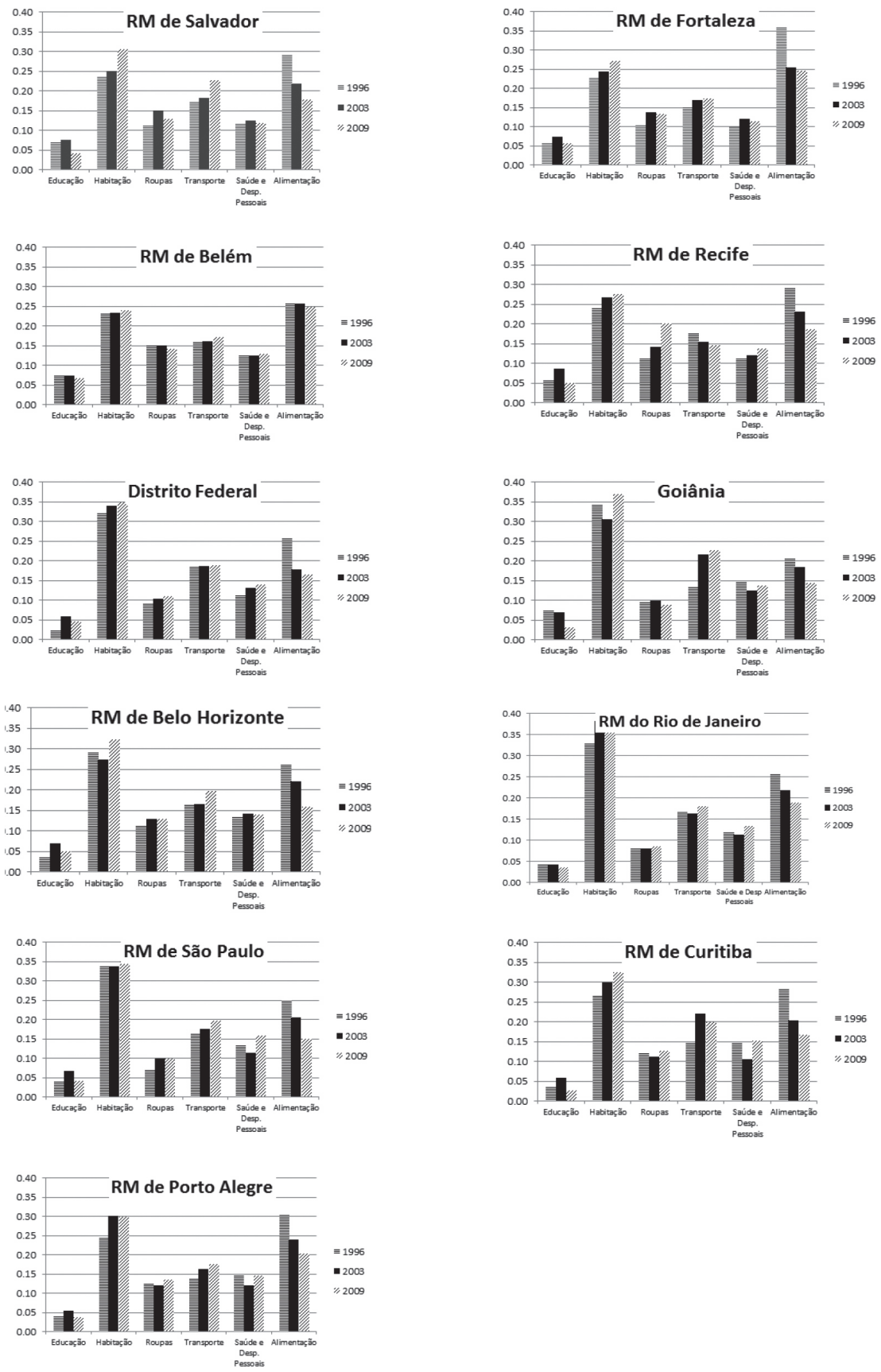

Figura 1 - Importância (peso) dos grupos de bens e serviços nos orçamentos familiares

Fonte: Dados da Pesquisa. 
Além dos pesos, é necessário conhecer os preços dos bens e serviços. Para o ano de 1996, o IBGE disponibiliza tabela detalhada de preços de alimentos e, para 1999, tabela com valores de aluguéis e itens não alimentares para cada uma das áreas. ${ }^{5}$ Nos anos em que a POF foi realizada, há informações sobre o valor gasto por domicílio e a quantidade adquirida de 22 dos itens considerados na pesquisa, permitindo o cálculo de preços implícitos, que foram considerados nos cálculos. Nos demais casos, foram utilizados os preços das tabelas de 1999. Esses vetores básicos de preços foram estendidos aos demais anos da série pelos procedimentos abaixo.

Para os preços implícitos, utilizaram-se informações sobre a variação de preços em cada área constantes do IPCA - Índice de Preços ao Consumidor Amplo, do IBGE. Os vetores de preços calculados nos anos da POF foram interpolados pelo seguinte procedimento. Seja $g^{I P C A}$ a taxa geométrica anual de crescimento do IPCA entre duas POF (por exemplo, entre 2003 e 2009) e $h^{P I}$ a taxa geométrica anual de crescimento dos preços implícitos das POF correspondentes. Com essas taxas, geraram-se duas séries geométricas anuais, uma para o IPCA e outra para os preços implícitos.

$$
\mathrm{IPCA}^{\mathrm{geo}} \mathrm{t}=\mathrm{IPCA}_{2003} * \mathrm{~g}^{\mathrm{IPCA}}(\text { entre } 2003 \text { e } \mathrm{t})
$$

Preço Implícito ${ }^{\mathrm{geo}} \mathrm{t}=$ Preço Implícito $_{2003}{ }^{*} \mathrm{~h}^{\mathrm{PI}}($ entre 2003 e $\mathrm{t})$

Em seguida, calcularam-se os desvios percentuais entre os IPCA efetivamente observados em cada ano e a série geométrica anual: $d^{I P C A}{ }_{t}=\left(I_{P C A} / I P_{C A}{ }_{t}^{\text {geo }}\right)$. Finalmente, introduziram-se esses desvios percentuais na série geométrica dos preços implícitos, gerandose os preços implícitos anuais utilizados no trabalho: Preço Implícito ${ }^{t}$ $=$ Preço Implícito ${ }_{\mathrm{geo}} * \mathrm{~d}_{\mathrm{t}}^{\mathrm{IPCA}}$. Com esse procedimento, mantivemos os preços implícitos conhecidos das POF e utilizamos a variabilidade do IPCA correspondente para interpolá-los. A oscilação das séries utilizadas é a mesma do IPCA (percentualmente), mas os valores são os dos preços implícitos. Isso foi feito entre 2003 e 2009. Para os anos posteriores a 2009, atualizaram-se os preços implícitos de 2009 pela variação do IPCA correspondente.

5 ftp://ftp.ibge.gov.br/Precos_Indices_de_Precos_ao_Consumidor/Sistema_de_Indices_de Precos ao Consumidor/ 
Como há problemas evidentes com os preços implícitos de alimentos de 1996, provavelmente porque as unidades de medida das quantidades variaram entre essa POF e as seguintes, retrocedemos os dados de 2003 pela variação do IPCA correspondente. No caso de aluguéis, os preços implícitos das POF revelaram-se problemáticos. Como o número de observações caiu muito da POF de 1996 para as demais, os valores médios apresentavam comportamento estranho entre anos e entre cidades. Assim, utilizamos como âncora os valores do vetor básico de preços 1999 disponibilizado pelo IBGE. Esses valores foram anualizados pelo índice de crescimento dos valores de aluguel das PNAD correspondentes às regiões metropolitanas e Brasília. Para Goiânia, adotamos a taxa de crescimento das áreas urbanas de Goiás. Em ambos os casos, consideramos imóveis similares - "imóvel com até 1 dormitório". ${ }^{6}$ Para 2000 e 2010, em que não há PNAD, e para 2014, utilizou-se a variação do IPCA. Para os demais itens foram considerados os preços da lista do IBGE como base, anualizando pelo IPCA correspondente.

\section{Níveis comparativos de custo de vida estimados}

Como exposto na metodologia, a Equação (1) foi usada para estimar os índices de preços. Primeiramente, para cada ano, estimou-se uma equação a partir dos preços e pesos de todos os itens da cesta de bens para calcular o índice geral. Em seguida, seis equações foram estimadas para calcular o índice de cada grande grupo de consumo. No total, 133 equações foram estimadas para os 19 anos do período considerado. ${ }^{7}$

Os resultados apresentados adiante revelam os níveis comparativos de custo de vida das principais regiões metropolitanas do país, mais DF e Goiânia durante o período 1996-2014. A Figura 2 apresenta os níveis relativos de custo de vida em três momentos distintos: média de 1996 a 1998, média de 2004 a 2006 e média de 2012 a 2014. As áreas estão dispostas em ordem decrescente do nível observado em 2012/14. Em cada ano, a média das áreas é a referência, e os níveis de cada RM ou cidade são expressos em percentagens em relação a

${ }^{6}$ Fizemos cálculo semelhante substituindo a variação dos valores de aluguel das PNAD pelas taxas de variação de aluguéis do IPCA. As séries de valores resultantes são muito próximas. 7 Os resultados das regressões estão disponíveis para consulta mediante solicitação aos autores. 
essa média. A ideia é fornecer informações sobre os níveis comparativos recentes e compará-los com os existentes no início do período analisado, oferecendo-se também uma informação intermediária.

Observa-se que o maior nível comparativo de custo de vida está no Distrito Federal, na faixa de $15 \%$ acima da média de todas as cidades consideradas, e com pequena alteração ao longo do período. Em segundo vem a RM de São Paulo, que inicia o período $12 \%$ acima da média e termina com 7\% acima. Um caso interessante é Goiânia, que no início do período estava bem abaixo da média ( $9 \%$ abaixo) e evoluiu celeremente para tornar-se tomar o terceiro lugar da RM do Rio de Janeiro já em 2004/06 e ampliando a diferença em 2014. A quarta RM mais cara em 2012/14 é o Rio de Janeiro, que se situava abaixo da média em 1996/98, mas cresceu comparativamente ao longo do período, para atingir 2\% acima da média em 2014. Levando em conta os níveis mais recentes, a RM de Porto Alegre situa-se em quinto lugar, tendo saído de $7 \%$ abaixo, superado ligeiramente a média no ano intermediário e retornado para abaixo da média em 2014 $(-2 \%)$. Todas as demais RM situam-se abaixo da média em todo o período. É notável, entretanto, a forte aproximação à média de Belo Horizonte (de 9\% abaixo para 2\% abaixo), Curitiba (de 10\% abaixo para $4 \%$ abaixo) e, em menor ritmo, Recife e Salvador. De outra parte, Fortaleza inicia $14 \%$ abaixo da média e torna-se progressivamente mais barata em relação às demais, terminando o período como a mais barata de todas as RM (19\% abaixo da média). Belém inicia o período na quarta posição ( $4 \%$ abaixo) e termina com $6 \%$ abaixo, na oitava posição.

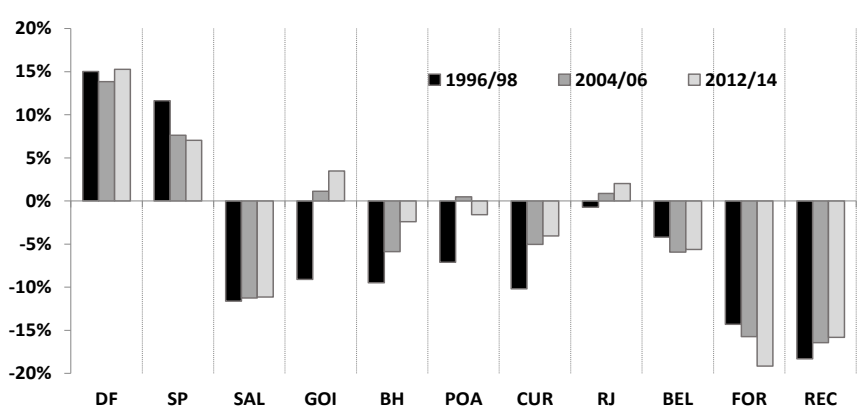

Figura 2 - Níveis Comparativos de Custo de Vida

Fonte: Dados da Pesquisa. 
As evoluções do custo de vida geral entre 1996 e 2014 estão apresentadas na Figura 3. Para facilitar a visualização, cada gráfico apresenta resultados para um grupo pequeno de cidades. Os resultados numéricos do índice geral e dos índices de grupos de bens e serviços são apresentados em apêndice. Na Figura 3 aparecem gráficos para o índice geral de custo de vida; em apêndice apresentam-se gráficos equivalentes para cada um dos grupos de bens e serviços considerados (Figuras 4 a 9). O gráfico superior direito reúne as áreas mais caras em 2014, DF, SP, Goiânia e RJ. Observa-se que esta última gravita em torno do eixo horizontal, situando-se ligeiramente acima dele. Isso revela que apresentou um nível de custo de vida próximo da média das 11 regiões metropolitanas consideradas, embora nos últimos anos tenha se afastado dela. A RM de São Paulo inicia o período próximo do nível de custo de vida do DF, mas apresenta evolução temporal inferior à da média das $11 \mathrm{RM}$, o que a leva a aproximar-se paulatinamente do patamar de 6\% acima. Já o DF, que inicia o período mais caro do que a RM de SP, acompanha esta última RM até 2002, passando a distanciar-se da média a partir de então. Em 2014, o nível de custo de vida dessa RM situava-se mais de $14 \%$ acima da média das 11 RM consideradas. A vizinha Goiânia apresenta evolução distinta de todas as demais, com crescimento constante ao longo do período, terminando como a terceira mais cara entre as demais áreas.

O gráfico inferior esquerdo apresenta a evolução de Belo Horizonte, Porto Alegre, Curitiba e Belém, todas com índices inferiores à média em 2014 e, com exceção de Curitiba, tendo permanecido abaixo da média em todos os anos. Também com a possível exceção dessa RM, mostraram crescimento constante a partir de 1999, aproximando-se da média das 11 áreas. Já o gráfico inferior direito mostra a evolução das três RM da região Nordeste, Salvador, Recife e Fortaleza, que se mantêm além de $10 \%$ abaixo da média, não havendo tendência evidente de mudança. 

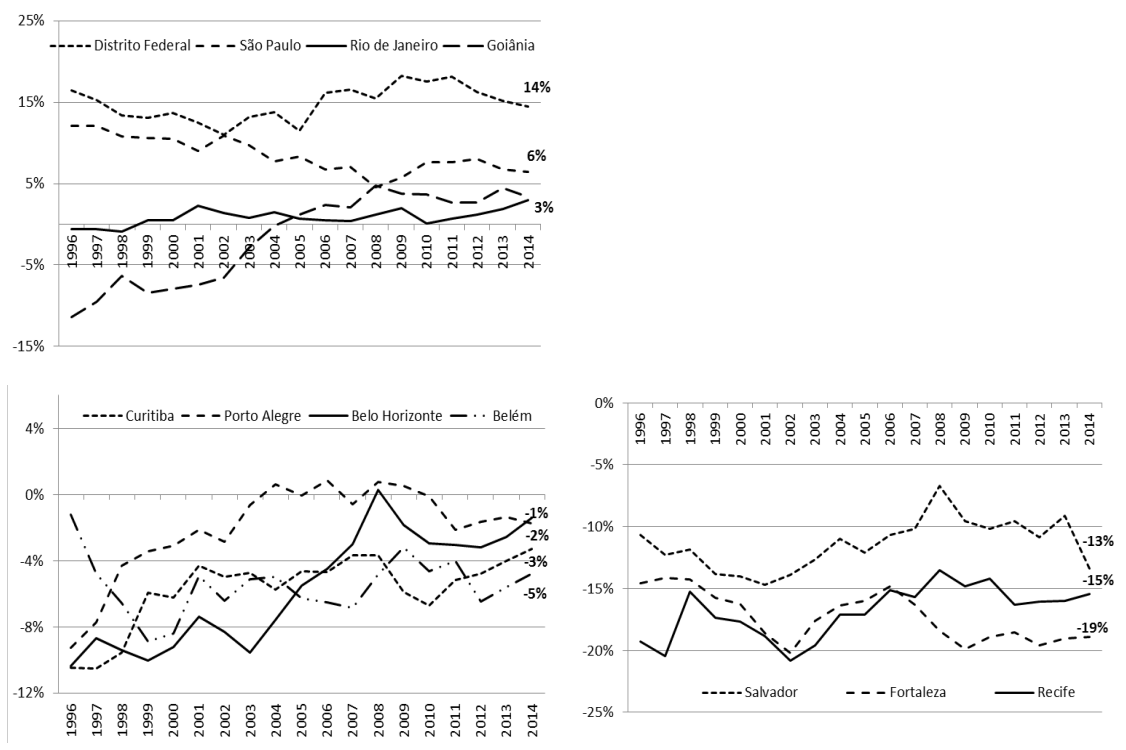

Figura 3 - Evolução comparativa do custo de vida das RMs - ÍNDICE GERAL Fonte: Dados da Pesquisa.

\section{Convergência de níveis de custo de vida entre as RM?}

A evolução dos níveis de custo de vida das regiões metropolitanas registrada acima parece indicar uma tendência de aproximação entre os níveis de custo de vida das RM. Para averiguar em que medida essa ideia procede, associamos o crescimento no nível de custo de vida ao longo do período ao nível no início do período. A Figura 4 apresenta no eixo vertical a variação nos níveis de custo de vida relativos ao longo do período e o eixo horizontal mostra os níveis relativos prevalecentes no seu início. Para evitar oscilações anuais eventuais, tomou-se a média dos três anos mais recentes (2012-2014) e dos três primeiros anos da série (1996-1999). A segunda RM mais cara no início do período, SP, decresceu relativamente, mas isso também ocorreu com Fortaleza e Belém. De outra parte, Recife, a segunda RM mais barata, cresceu relativamente no período. As RM de Curitiba, Belo Horizonte e Porto Alegre apresentaram crescimentos, mas o destaque, como apontado anteriormente, fica com Goiânia, que apresentou o maior crescimento. Uma regressão desses cresci- 
mentos contra os respectivos níveis iniciais indica que não se pode dizer que não tenha havido convergência nos níveis de custo de vida, mas certamente o ritmo em que isso ocorre é bastante lento.

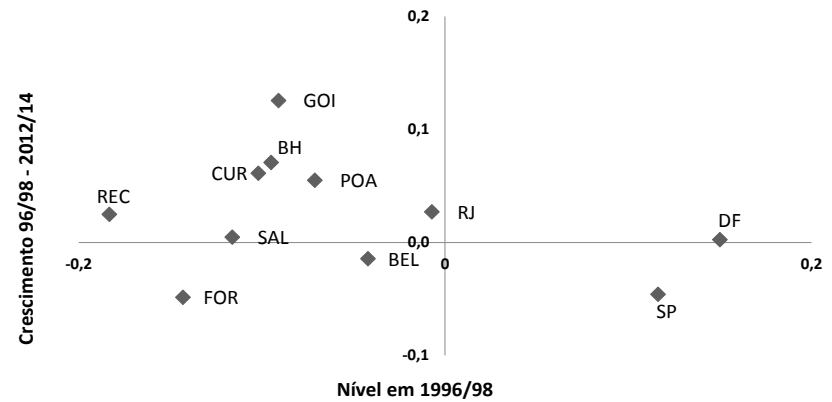

Figura 4 - Evolução e Níveis Iniciais de Custo de Vida

Fonte: Dados de Pesquisa.

Nos gráficos da Figura 5 a mesma informação é desagregada por grupos de bens e serviços, permitindo apontar possíveis regularidades internamente a cada grupo. Os grupos Habitação, Alimentos e Transporte apresentam tendência decrescente, particularmente este último. Ou seja, nesses casos, as cidades inicialmente mais caras tiveram seus custos crescendo menos e cidades mais baratas, crescendo mais. Já os grupos Saúde e Despesas Pessoais e Vestuário apresentam tendência crescente, indicando que cidades mais caras tornaram-se ainda mais caras ao longo do tempo. O grupo Educação não apresenta tendência evidente. No grupo transportes a correlação negativa é mais evidente, com as três grandes RM apresentando crescimento de custo inferior à média, em que pese terem níveis relativos iniciais maiores (com exceção do RJ). Provavelmente, a homogeneização das estruturas de oferta desse tipo de serviço (mesmas empresas competindo em diferentes cidades, homogeneização das condições salariais e de segurança etc.) tenha feito com que, relativamente, o custo nas cidades menores tenha crescido mais rápido do que nos grandes centros. 

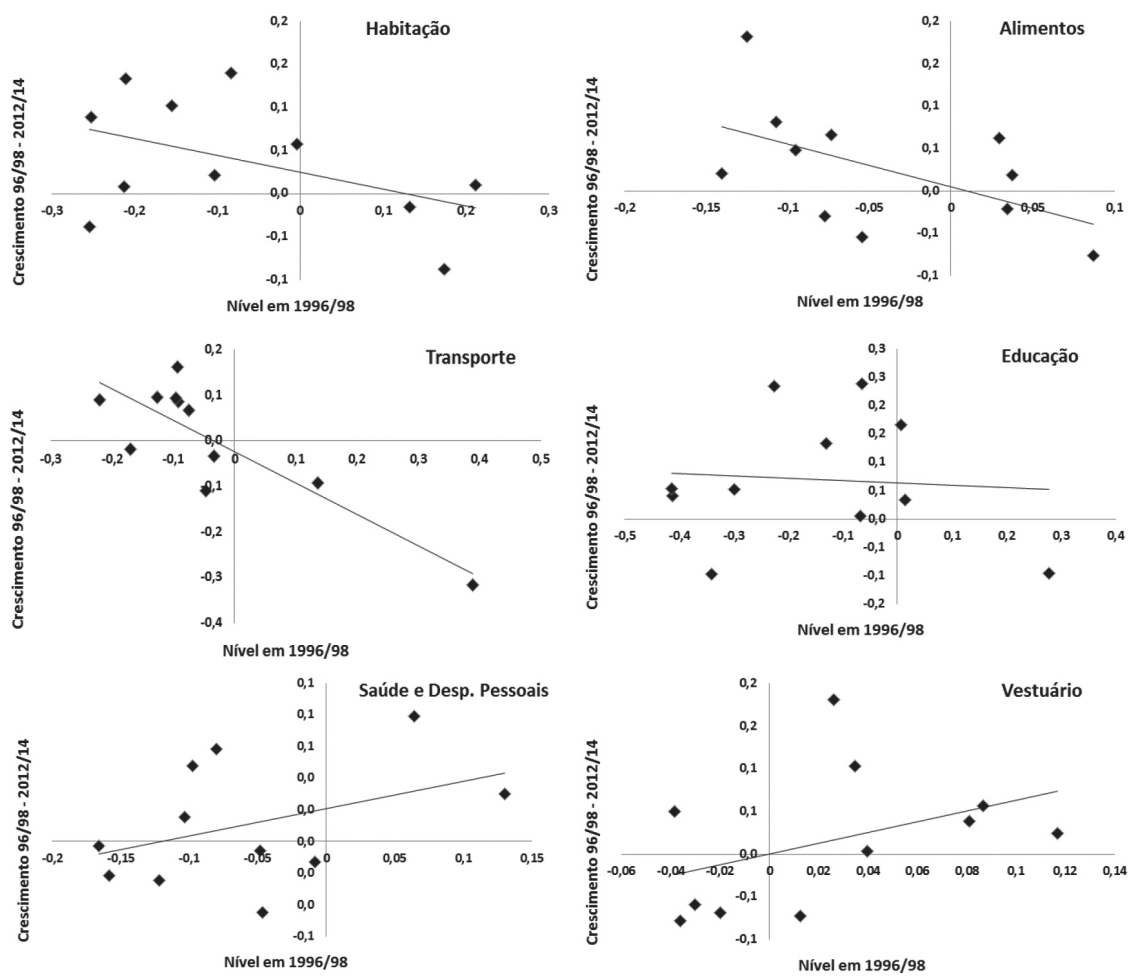

Figura 5 - Evolução e Níveis Iniciais de Custo de Vida por Grupo (Linhas inseridas para fins ilustrativos apenas).

Fonte: Dados da Pesquisa.

\section{Evolução da renda, paridade do poder de compra e desigual- dade regional}

Na Figura 6 apresentam-se as evoluções das rendas per capita relativas à média nacional, com base nos dados da PNAD (todas as fontes). As regiões metropolitanas são distribuídas em dois grupos distintos, sendo que os gráficos superiores retratam as posições das RM das regiões Norte e Nordeste; os inferiores retratam as RM das regiões Sudeste e Sul, além de Brasília. Nos gráficos da esquerda aplicou-se um deflator nacional, idêntico a todas as RM (IPCA); nos gráficos da direita aplicaram-se adicionalmente os índices calculados neste trabalho, que levam em conta os níveis específicos de custo de vida e sua evolução. Nota-se um deslocamento para cima no caso das 
RM do norte e nordeste, e um deslocamento para baixo no caso das demais, como esperado. Por exemplo, a RM de Fortaleza apresenta em 2013 uma renda nominal que é 63\% da renda média nacional; quando se leva em conta que essa RM é mais barata do que a média nacional, sua posição relativa sobe para $92 \%$ da média nacional. No mesmo ano, Brasília tem um nível de renda 60\% superior à média nacional; quando considerado o alto custo de vida dessa RM, sua posição relativa cai para 39\% acima da média nacional.

Renda Real (Deflator Nacional)
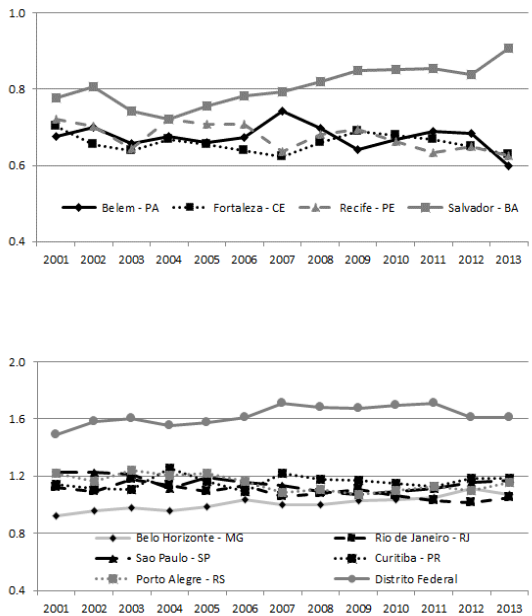

PPP (Deflator Nacional + Regional)
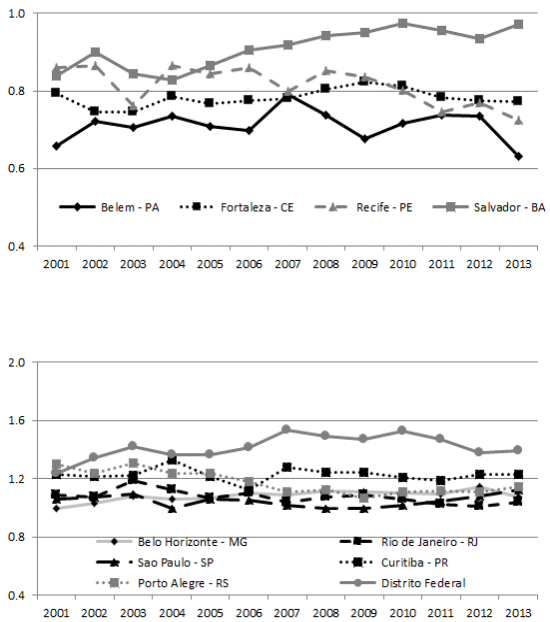

Figura 6 - Evolução da Renda Real e da PPP Regional (médias nacionais = 1) Fonte: Dados da Pesquisa.

A Figura 7 apresenta os coeficientes de variação das rendas per capita, ponderados pela população de cada RM, indicador esse que revela a dispersão das rendas per capita em cada momento do tempo. Nota-se que a dispersão das rendas sem deflacionamento regional é levemente maior do que quando se considera a PPP regional, como era de se esperar, à luz dos resultados já apresentados e comentados neste trabalho. Em termos de evolução, ambas crescem, indicando que a dispersão de renda per capita entre as RM aumentou ao longo do período. A linha pontilhada descreve a evolução do relativo entre os dois indicadores até 2013, mostrando estabilidade e sem tendência entre os índices, conforme o esperado. 


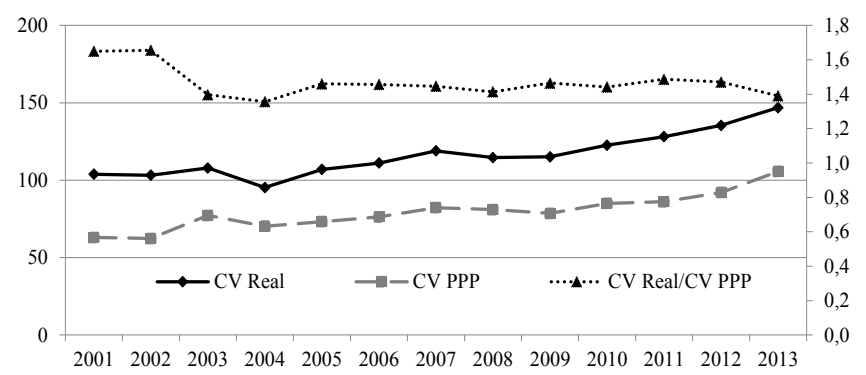

Figura 7 - Coeficiente de Variação Ponderado (CV)

Fonte: Dados da Pesquisa.

Finalmente, calculamos a desigualdade entre as RM nos anos em que as POF foram aplicadas através do índice de Gini, com os resultados expostos na Tabela 1 . Nota-se que a desigualdade regional das rendas per capita deflacionadas pelos índices apresentados neste trabalho é sempre menor do que a calculada sem levar em conta as diferenças em níveis de custo de vida. A desigualdade regional de poder aquisitivo indicada pelo índice de Gini regional é 19\% inferior à desigualdade calculada sem levar em conta os índices regionais em 1996, e 28\% inferior em 2003 e 2009. Trata-se de diferenças importantes, que indicam que a desigualdade de acesso a bens e serviços entre as populações dessas áreas não é tão pronunciada quanto a desigualdade de renda indica. Esse é um bom exemplo da utilidade dos indicadores de níveis de custo de vida regionais em análises de diferenciais das condições sociais entre distintas áreas, já que ignorar essa dimensão levaria a superestimar a extensão da desigualdade.

Tabela 1 - Desigualdade entre as RM - Gini Regional

\begin{tabular}{cccc}
\hline Ano & Rendas Nominais - A & Rendas Reais $\left(^{*}\right)-\mathrm{B}$ & $(\mathrm{B} / \mathrm{A})$ \\
\hline 1996 & 0,1453 & 0,1177 & 0,81 \\
2003 & 0,1714 & 0,1241 & 0,72 \\
2009 & 0,1098 & 0,0795 & 0,72 \\
\hline
\end{tabular}

(*) Aplicando-se aos valores nominais os índices regionais apresentados neste trabalho. Fonte: Dados da pesquisa.

\section{Considerações finais}

Neste trabalho foram calculados níveis de custo de vida para as nove regiões metropolitanas oficiais brasileiras, além das cidades de Goiânia e o Distrito Federal, no período de 1996 a 2014. Os níveis 
relativos de custo de vida não se alteraram significativamente no período, com DF, São Paulo e Rio de Janeiro mantendo os maiores níveis. Goiânia saltou de níveis abaixo da média no início do período para suplantar o Rio de Janeiro como terceira cidade mais cara do país. Porto Alegre seguiu caminho parecido mas terminou o período ainda abaixo da média. No outro extremo, Salvador, Fortaleza e Recife continuam sendo as RM com menores níveis comparativos de custo de vida. Todavia, Fortaleza vem paulatinamente afastando-se da média, enquanto Salvador vem dela se aproximando, caminho também trilhado por Belo Horizonte. Analisando-se a evolução dos níveis de relativos de custo de vida, há tênues indícios de que as diferenças vêm diminuindo ao longo do período. A análise dos grupos de bens e serviços indica que Transportes e, em menor grau, Habitação e Alimentos, apresentaram convergência, com as cidades inicialmente mais caras crescendo menos do que as demais. Já Vestuário e Saúde e Despesas Pessoais apresentaram divergência.

Cabe salientar que a análise aqui desenvolvida sempre considera níveis relativos de custo de vida. Reduções no nível relativo de custo de vida de determinada cidade não significam que tenha havido redução em nível absoluto. Tal resultado é causado por aumentos menores do que nas outras cidades, associados a mudanças na importância de cada grupo nos orçamentos familiares. Assim, mesmo em uma situação de inflação alta, uma cidade pode experimentar redução no seu nível relativo de custo de vida. Tudo depende dos ritmos diferenciados da inflação nas diferentes cidades.

Os resultados aqui dispostos apresentam utilidade muito grande em estudos regionais, posto que oferecem a possibilidade de estudar a desigualdade em níveis de renda per capita, por exemplo, descontando as diferenças em níveis de custo de vida. Isso proporciona uma melhor análise da desigualdade de bem estar entre as populações das distintas regiões brasileiras.

\section{Referências}

ATEN, B. Estimates of State and Metropolitan Price Parities for Consumption Goods and Services in the United States, 2005, Bureau of Economic Analysis, Working Paper, October 2008.

Estimates of State Price Levels for Consumption Goods and Services: A First Brush", Bureau of Economic Analysis, Working Paper, 2007. 

2005.

. "Report on Interarea Price Levels", Bureau of Economic Analysis, Working Paper, November

ATEN, B., D'SOUZA, R. D. Estimates of State and Metropolitan Price Levels for Consumption Goods and Services in the United States, Survey of Current Business, Bureau of Economic Analysis, November, 2008.

ATEN, B., FIGUEROA, E.; MARTIN, T. How can the American Community Survey (ACS) be used to improve the imputation of Owner-Occupied Rent Expenditures? Bureau of Economic Analysis, Working Paper, February 2012.

. Estimating the Price of Rents in Regional Price Parties, Bureau of Economic Analysis, Working Paper, October, 2011a.

Notes on Estimating the Multi-Year Regional Price Parities by 16 Expenditure Categories: 2005-2009 Bureau of Economic Analysis, Working Paper, $2011 \mathrm{~b}$.

ATEN, B., REINSDORF, M. Comparing the Consistency of Price Parities of Regions of the U.S. in an Economic Framework Approach, Bureau of Economic Analysis, Working Paper, August 2010.

AZZONI, C. R., GUILHOTO, J., SILVEIRA, F. G., MENEZES, T., HADDAD, E. A., HAZEGAWA, M. Commodity price changes and their impacts on poverty in Developing Countries: the Brazilian case. Studies in Regional Science / Chiikigaku kenky, v.39, p.131 - 147, 2009.

AZZONI, C. A.; CARMO, H. E.; MENEZES, T. Comparação da Paridade do Poder de Compra entre Cidades: Aspectos Metodológicos e Aplicação ao Caso Brasileiro. Pesquisa e Planejamento Econômico, 33(1), 91-126, 2003.

AZZONI, C. A.; CARMO, H. E.; MENEZES, T. Índice de Custo de Vida Comparativo para as Principais Regiões Metropolitanas Brasileiras: 1981-1999. Estudos Econômicos, 30(1), 165-186, 2000.

DEATON, A. E MUELlBAUER, J., An Almost Ideal Demand System,. The American Economic Review. v.70, n. 3, p. 312-326, June 1980.

DELLER, STEVEN C., SHIELDS, M.; TOMBERLIN, D. Price Differentials and Trends in State Income Levels: A Research Note. Review of Regional Studies, v.26, pp99-113, 1996.

HESTON, A. E LIPSEY, R. International and Comparisons of Income, Output, and Prices, 1999, 1-530. National Bureau of Economic Research, University of Chicago Press, Chicago, 1999, p. 211-226.

HESTON, A E SUMMERS, R., International price and quantity comparison: potentials and pitfalls. American Economic Review v.86, Iss. 1, May 1996, pp.20-24

Instituto Brasileiro de Geografia e Estatística. Pesquisa de Orçamentos Familiares 2002-2003: primeiros resultados. Rio de Janeiro: IBGE, 276p, 2004.

. Pesquisa de Orçamentos Familiares 2008-2009: Despesas, Rendimentos e Condições de Vida. Rio de Janeiro: IBGE, 222p, 2010.

JOHNSTON, R.; MCKINNEY, M.; STARK, T. Regional Price Level Variation and Real Household Incomes in United Kingdom, 1979/1980-1993. Regional Studies, 30(6), pp. 567-578, 1996.

KOKOSKI, M. Quality Adjustment of Price Index. Monthly Labor Review, Bureau of Labor Statistics, Division of Price Index Number Research, December 1993.

KOKOSKI, M.; MOULTON, B. Experimental interarea consumer price index: estimation and aggregation. Monthly Labor Review Bureau of Labor Statistics, Division of Price Index Number Research, November 1996.

KRAVIS, B.; HESTON A.; Summers, R. World Product and Income: international comparations of real gross product. Washington DC: The World Bank, 1982.

MCMAHON, W. Geographical Cost of Living Differences: An Update. Real State Economics, 19(3), pp. 426-450, 1991.

MENEZES, T.; AZZONI, C. R.; SILVEIRA, F. G. Demand Elasticities for Food Products in Brazil: A Two-Stage Budgeting System. Applied Economics, 40, 2557-2572, 2008. 
MENEZES, T.; AZZONI, C. R. Estimação de Estruturas de Ponderação para Cálculo de Custo de Vida em Cidades Brasileiras. Universidade de São Paulo, mimeo, 2003.

MENEZES, T. A., AZZONI, C. R. E MOREIRA, G. R. C. Diferenças em gastos com aluguel entre estados, tipos de área e níveis de renda familiar no Brasil. In: Fernando Gaiger Silveira; Luciana Mendes Santos Servo; Tatiane Almeida de Menezes; Sérgio Francisco Piola. (Org.). Gasto e consumo das famílias brasileiras contemporâneas. Brasília: IPEA, v. 2, p. 329-346, 2007.

MOROLlÓN, F. R., NAVAMUEL, E. L. E VÁZQUES, E. F. Efectos de los incrementos en el coste de vida sobre el mapa de la pobreza en España. Papeles de economía española, No. 138, pp. 114-128, 2013.

NAVAMUEL, E. L., PAREDES, D. E VÁZQUES, E. F. A true cost of living index for Spain using a microeconomic approach and censored data. Documentos de Trabajo en Economia y Ciencia Regional 26, Universidad Catolica del Norte, Chile, Department of Economics, 2012 https://sites. google.com/a/ucn.cl/wpeconomia/archivos/WP2012-12.pdf

NERI, M. The Decade of Falling Income Inequality and Formal Employment Generation in Brazil. In: OECD (org), Tackling Inequalities in Brazil, China, India e South Africa: The Role of Labour Market and Social Policies. Paris: OECD Publishing, 2010.

SAVEDOFF, W. D., Os diferenciais regionais de salários no Brasil: segmentação versus dinamismo da demanda. Pesquisa e Planejamento Econômico, 20(3) p.521-556, 1990.

WALDEN, M. How Much Income Variation “Really” Exists Within a State? Review of Regional Studies, 27(3), pp. 237-150, 1997.

\section{Apêndice}

\section{Composição dos grupos}

\begin{tabular}{|c|c|c|c|c|}
\hline \multirow{3}{*}{ Educação } & \multicolumn{3}{|c|}{ Mensalidade e Matricula (1 Grau) } & Ônibus Urbano \\
\hline & \multicolumn{3}{|l|}{ Cursos de Inglês } & Taxi \\
\hline & \multicolumn{2}{|c|}{ Caneta Esferográfica (Unidade) } & Transporte & Conserto de Automóvel (hora) \\
\hline \multirow{8}{*}{ Habitação } & & & & Gasolina Comum (Litro) \\
\hline & \multicolumn{2}{|l|}{ Imóvel com 1 Quarto } & & Álcool (Litro) \\
\hline & \multicolumn{3}{|l|}{ Condomínio } & \\
\hline & \multicolumn{2}{|c|}{ Taxa de Água e Esgoto } & & Analgésico e Antitérmico (4 compri \\
\hline & \multicolumn{2}{|l|}{ Gás de Botijão } & & Consulta Médica \\
\hline & \multicolumn{2}{|l|}{ Luz } & Saúde e Despesas & Obturação \\
\hline & \multirow{2}{*}{\multicolumn{2}{|c|}{ Telefone Fixo }} & Pessoais & Desodorante \\
\hline & & & & Sabonete \\
\hline \multirow{3}{*}{ Roupas } & \multirow{3}{*}{\multicolumn{2}{|c|}{$\begin{array}{l}\text { Calça Masculina } \\
\text { Calça Feminina } \\
\text { Tênis }\end{array}$}} & & Corte de Cabelo \\
\hline & & & & \\
\hline & & & & \\
\hline \multirow{9}{*}{ Alimentos } & Arroz & \multicolumn{2}{|l|}{ Ovos } & \\
\hline & Feijão-Preto & \multicolumn{2}{|l|}{ Leite } & \\
\hline & Macarrão com ovos & \multicolumn{2}{|c|}{ Biscoito } & \\
\hline & Batata-Inglesa & \multicolumn{2}{|c|}{ Pão-Francé } & \\
\hline & Cebola & \multicolumn{2}{|c|}{ Óleo de soj } & \\
\hline & Cenoura & \multicolumn{2}{|c|}{ Refrigerant } & \\
\hline & Carne de Porco & \multicolumn{2}{|c|}{ Café Moídı } & \\
\hline & Contra-filé & \multicolumn{2}{|c|}{ Sal Refinac } & \\
\hline & Frango & \multicolumn{2}{|c|}{ Lanche } & \\
\hline
\end{tabular}




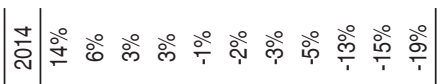

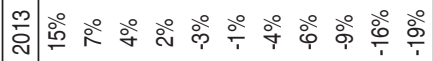

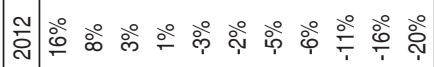

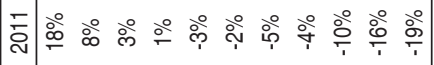

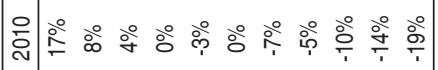

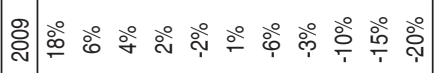

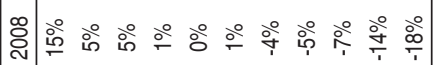

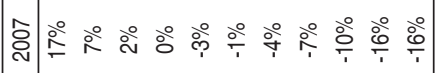

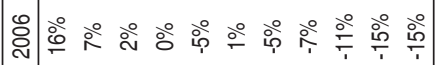

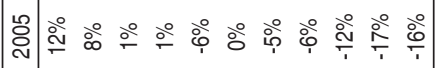

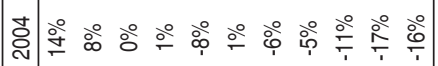

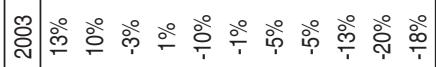

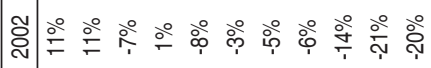

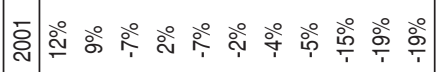

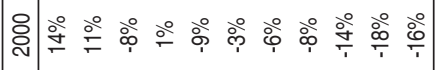

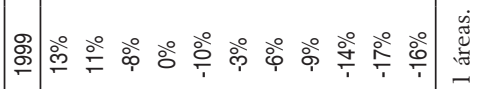

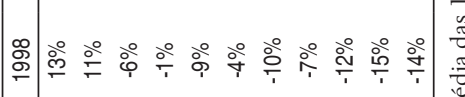

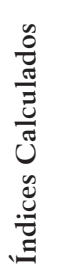

ㄴ.

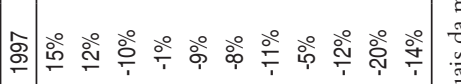

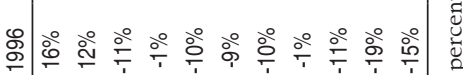
嵒

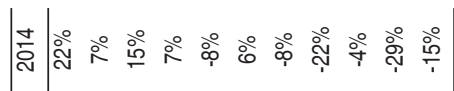

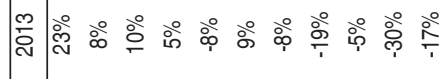

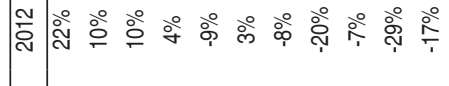

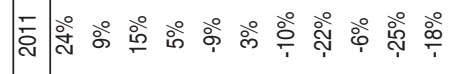

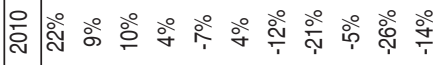

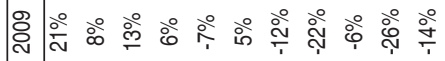

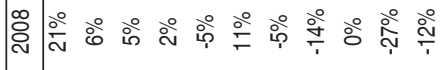

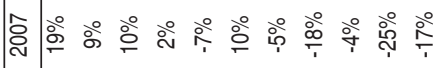

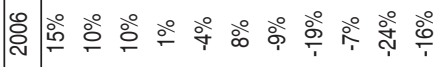

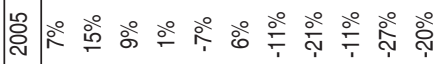

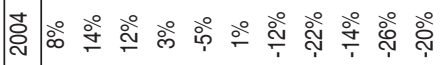

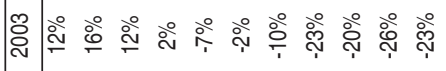

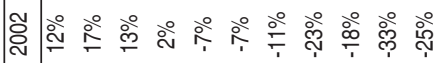

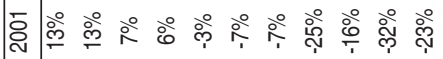

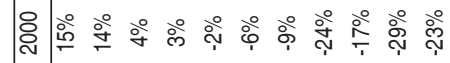

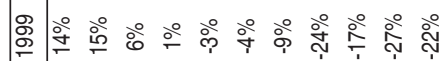

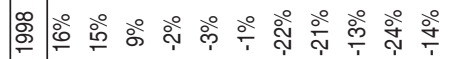

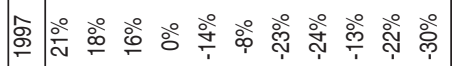

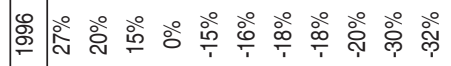
丞 


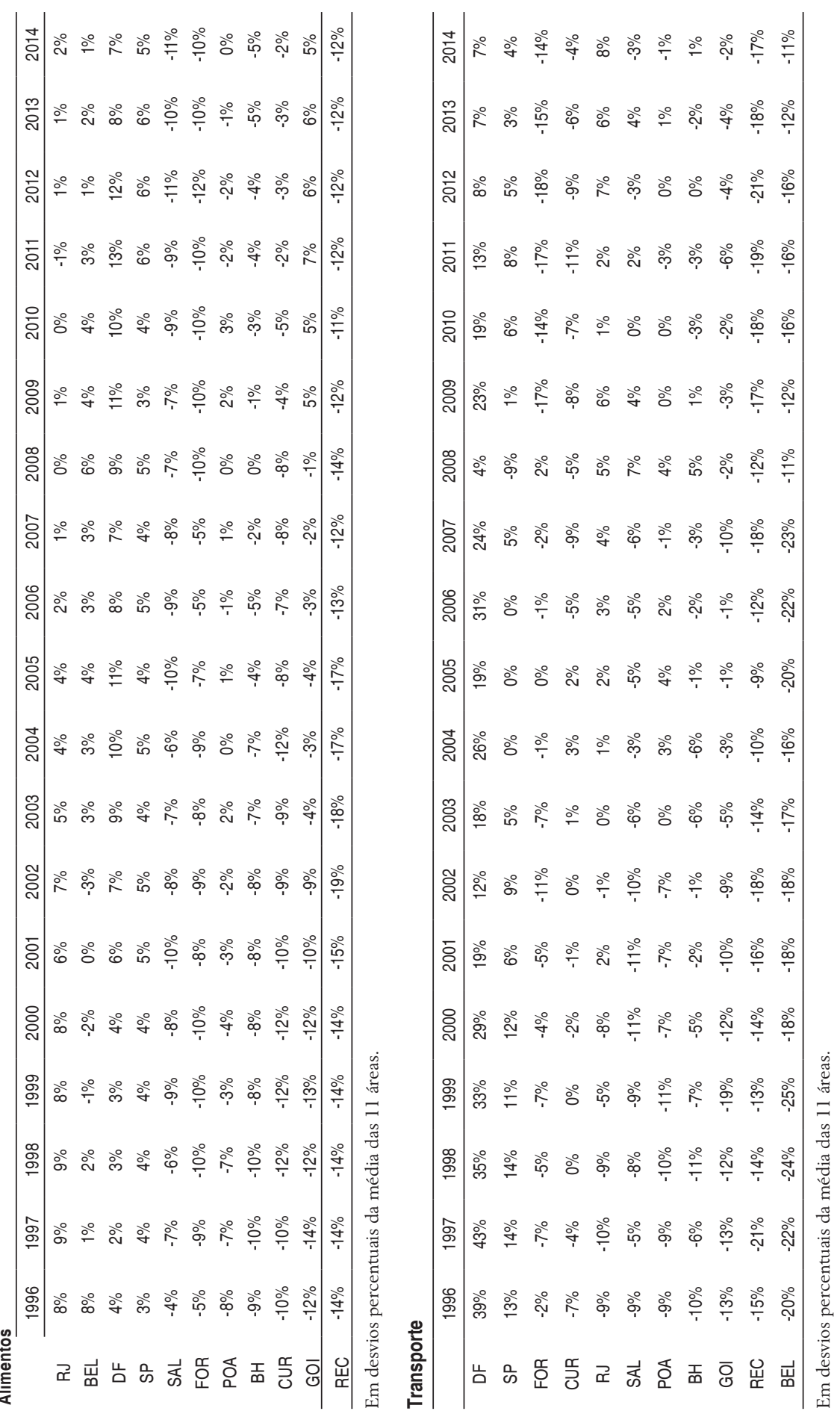




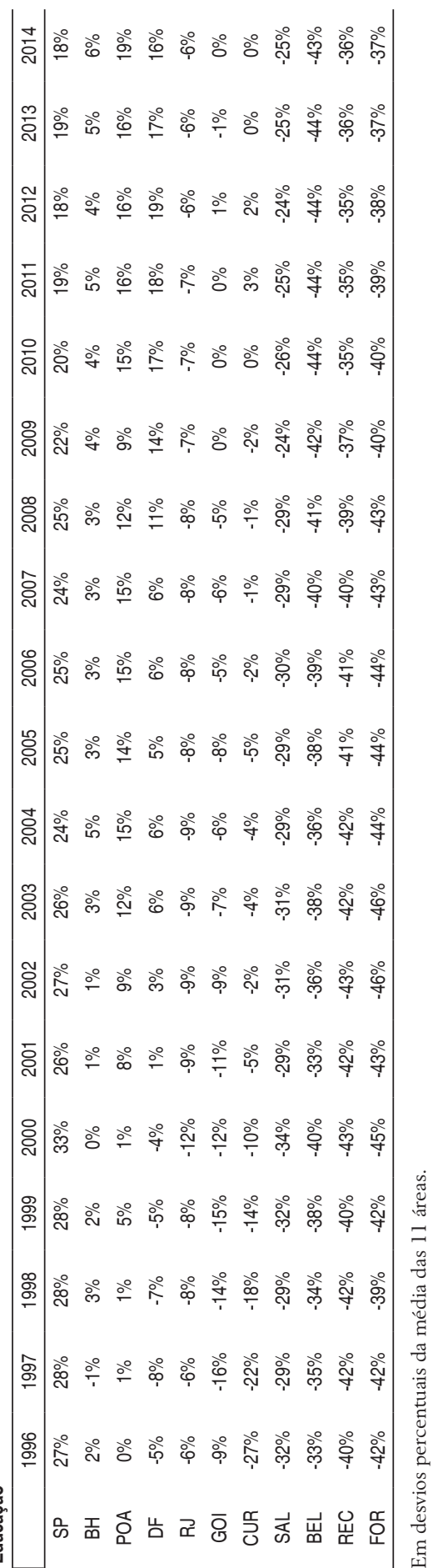

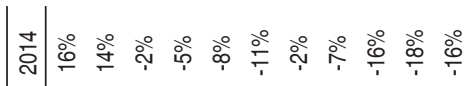

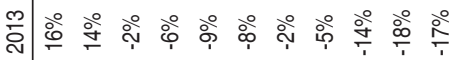

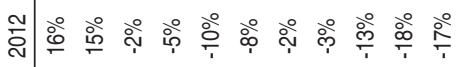

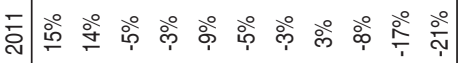

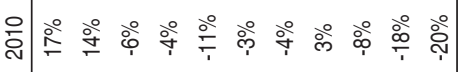

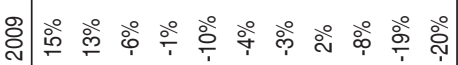

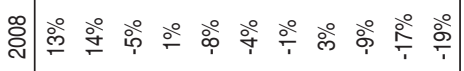

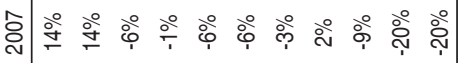

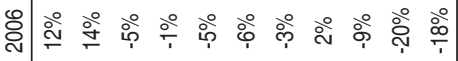

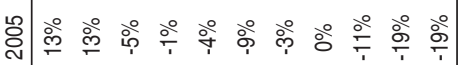

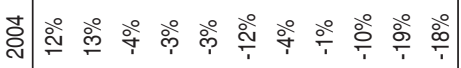

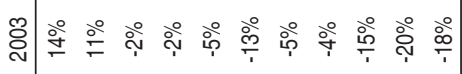

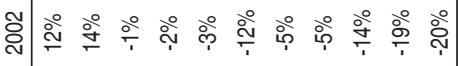

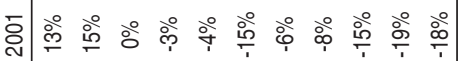

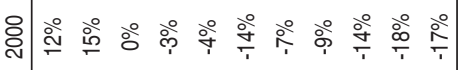

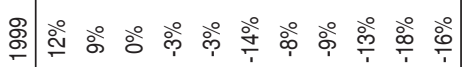

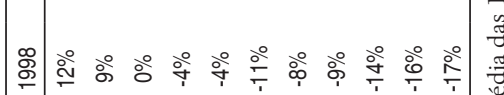

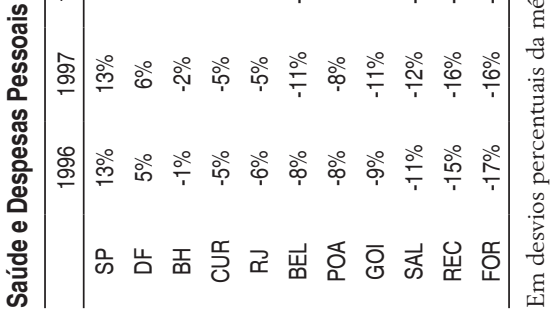




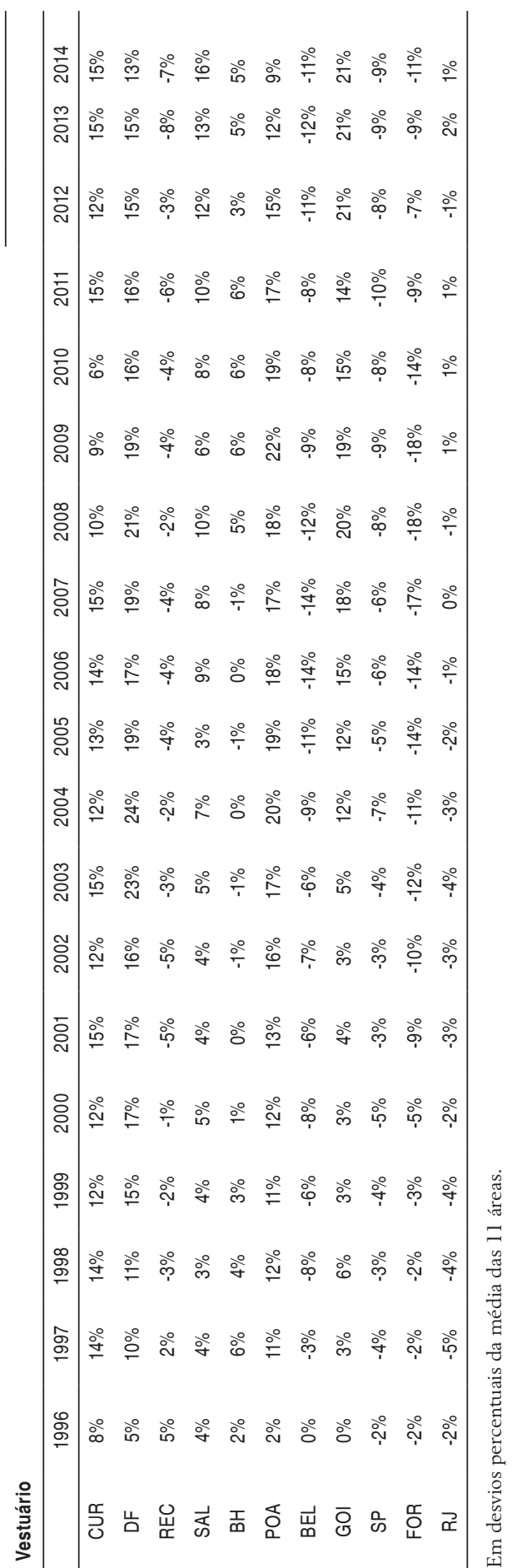

